\title{
Effect of osimertinib treatment on lung adenocarcinoma with squamous cell transformation harboring the T790M mutation: A case report and literature review
}

\author{
FUMIHIRO YAMAGUCHI, EISUKE KATO, AYA WAKABAYASHI and YUSUKE SHIKAMA \\ Department of Respiratory Medicine, Showa University Fujigaoka Hospital, Yokohama, Kanagawa 227-8501, Japan
}

Received October 26, 2018; Accepted May 30, 2019

DOI: $10.3892 / \mathrm{mco} .2019 .1880$

\begin{abstract}
We herein report a case of squamous cell transformation combined with the epidermal growth factor receptor $(E G F R)$ mutation T790M associated with acquired resistance to EGFR tyrosine kinase inhibitors (EGFR-TKIs) in a 73-year-old male patient diagnosed with stage IVA lung adenocarcinoma. Gene alterations were analyzed by collecting and studying pleural effusion at the time of diagnosis. Examination revealed an exon 19 deletion in the EGFR gene. Following treatment with the second-generation EGFR-TKI afatinib, squamous cell carcinoma was identified by performing a re-biopsy of the recurrent site. Although the levels of cytokeratin 19 fragment, which is a tumor marker for the follow-up of squamous cell carcinoma, were increased at that point, the levels of carcinoembryonic antigen, a marker particularly associated with adenocarcinoma, remained within normal limits. The T790M mutation and the original exon 19 deletion were detected simultaneously. The patient received treatment with the third-generation EGFR-TKI osimertinib, achieving a good clinical response. These findings suggest that osimertinib is beneficial for lung adenocarcinoma patients with squamous cell transformation harboring the T790M mutation.
\end{abstract}

\section{Introduction}

The use of epidermal growth factor receptor-tyrosine kinase inhibitors (EGFR-TKIs) has been approved for the treatment of advanced non-small-cell lung carcinoma (NSCLC) with $E G F R$-activating mutations. Although their efficacy is

Correspondence to: Dr Fumihiro Yamaguchi, Department of Respiratory Medicine, Showa University Fujigaoka Hospital, 1-30 Fujigaoka, Aoba-ku, Yokohama, Kanagawa 227-8501, Japan

E-mail: f_y@med.showa-u.ac.jp

Abbreviations: EGFR, epidermal growth factor receptor; TKI, tyrosine kinase inhibitor; NSCLC, non-small-cell lung carcinoma; CEA, carcinoembryonic antigen; CYFRA, cytokeratin 19 fragment; ROS, reactive oxygen species

Key words: T790M mutation, lung adenocarcinoma, squamous cell transformation, adenosquamous carcinoma, osimertinib well-established, almost all patients treated with EGFR-TKIs eventually develop resistance to these agents. Of those patients, $60 \%$ carry the missense mutation T790M in EGFR (1), which may occur concurrently with human epidermal growth factor receptor 2 (HER2) mutation, MET amplification, or small-cell transformation. Hence, the T790M mutation plays an important role in acquired resistance to treatment with EGFR-TKIs. In 2015, the third-generation EGFR-TKI osimertinib was approved for the treatment of NSCLC harboring the T790M mutation in patients with disease progression following therapy with first- or second-generation EGFR-TKIs. Therefore, re-biopsy may be used to detect the mutation responsible for the development of resistance in such patients.

The present case report details the occurrence of squamous cell transformation in combination with the T790M mutation and the efficacy of osimertinib in a patient with lung adenocarcinoma.

\section{Case report}

A 73-year-old male Japanese patient, with a smoking history of 75 pack-years, was referred to the Department of Respiratory Medicine of Showa University Fujigaoka Hospital (Yokohama, Japan) with dyspnea on effort. A chest X-ray revealed severe pleural effusion in the left lung and a chest computed tomography scan revealed a primary tumor $(77 \mathrm{~mm}$ in greatest diameter) in the left upper lobe. In addition, positron emission tomography detected a mass in the left adrenal gland. There was no evidence of brain or bone metastasis. The levels of the tumor markers carcinoembryonic antigen (CEA) and cytokeratin 19 fragment (CYFRA) were increased; (CEA: $17.7 \mathrm{ng} / \mathrm{ml}$; normal range: 0-5.0 ng/ml, CYFRA: $11.4 \mathrm{ng} / \mathrm{ml}$; normal range: $0-3.5 \mathrm{ng} / \mathrm{ml}$; Fig. 1). Adenocarcinoma cells were detected in the pleural effusion. Immunohistochemical analysis was not possible due to the limited number of cancer cells. Transbronchial lung biopsy from the left upper lobe did not demonstrate malignant findings. The patient was diagnosed with stage IVA lung adenocarcinoma, and cytology examination using a specimen from the pleural effusion revealed the presence of an EGFR-activating mutation (deletion E746-A750 in exon 19). Hence, treatment with the second-generation EGFR-TKI afatinib (40 mg) was initiated as first-line therapy. 


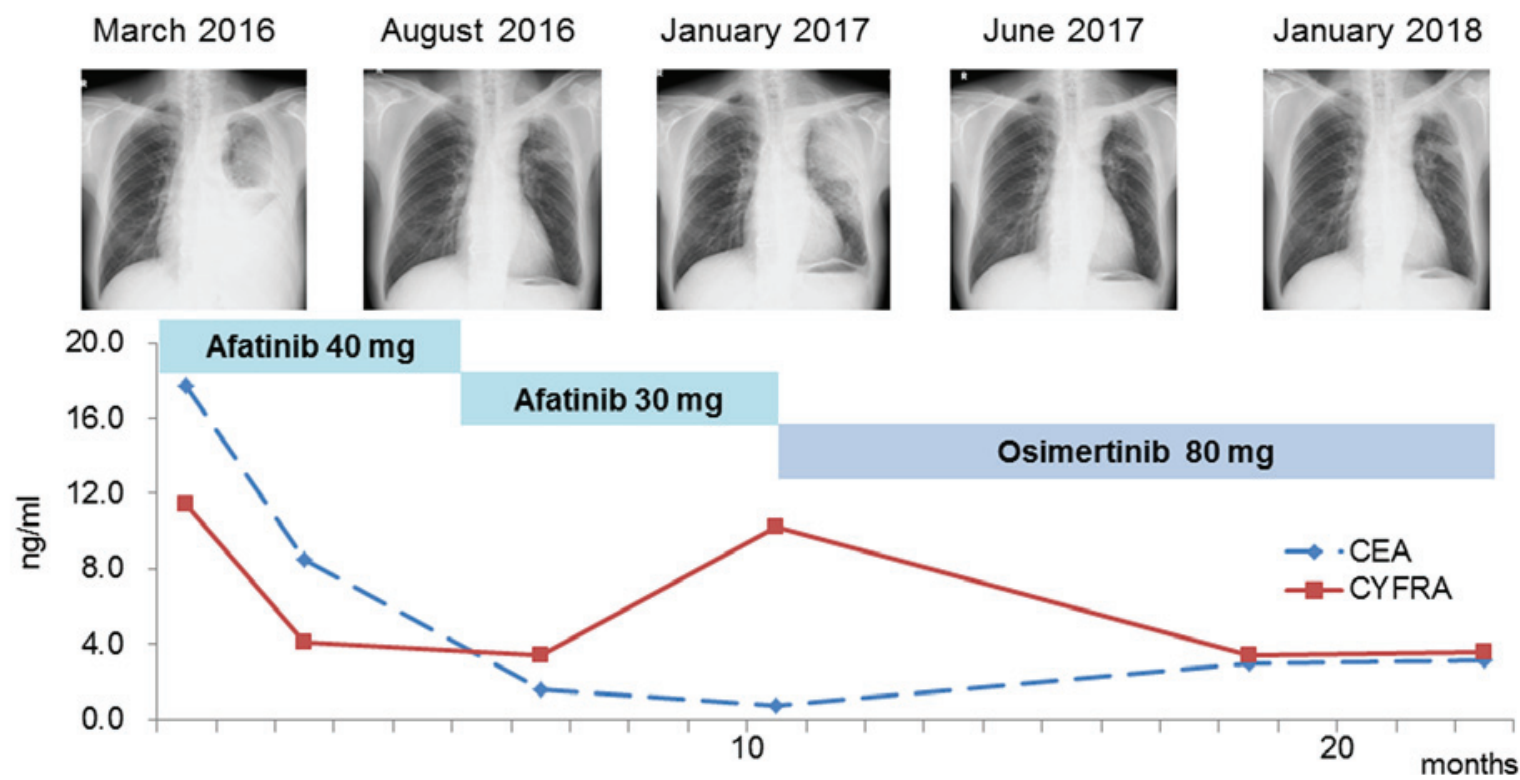

Figure 1. Clinical course of the present case. Chest X-rays show marked regression of the tumors following afatinib and osimertinib treatment. Line chart demonstrates the EGFR-TKI effect on tumor marker levels. EGFR, epidermal growth factor receptor; TKI, tyrosine kinase inhibitor; CEA, carcinoembryonic antigen; CYFRA, cytokeratin 19 fragment.

$\mathrm{H} \& \mathrm{E}$

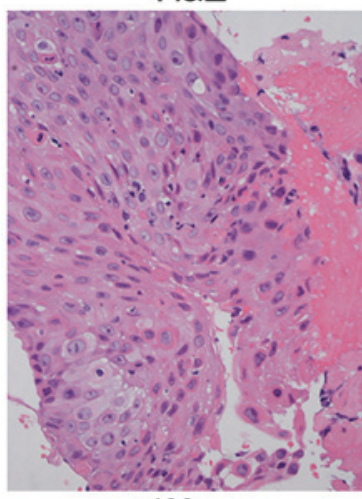

$x 400$ p40

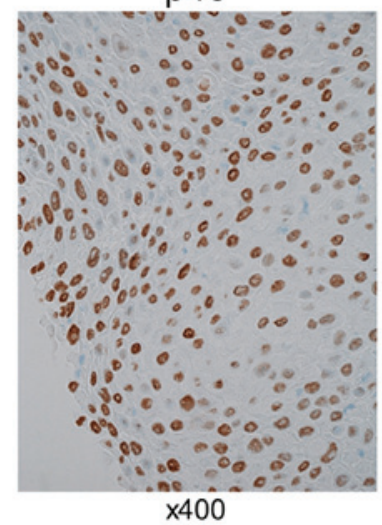

Figure 2. Pathological findings. Hematoxylin and eosin (HE) staining and p40 immunostaining of a re-biopsy specimen demonstrated a predominantly squamous cell carcinoma histology.

Gene alterations in lung carcinoma were analyzed by collecting and examining pleural effusion at the time of diagnosis. DNA was extracted from cell pellets using the QIAmp DNA Mini kit (Qiagen, Valencia, CA, USA). The PCR-Invader (BML Inc., Tokyo, Japan) method was performed to detect EGFR mutations. Re-biopsy specimens were also analyzed in a similar manner. Official approval for gene analysis was obtained in advance from the Ethics Committee for Genomic Research at Showa University (approval no. 113) and the patient provided written informed consent.

At 5 months after initiation of afatinib treatment, the primary tumor site had almost disappeared, without observed recurrence. In addition, the levels of CEA and CYFRA returned to normal. The treatment course exacerbated the skin lesions at grade 3 according to the Common Terminology Criteria for Adverse Events version 4.0 (2), and the dosage of afatinib was adjusted to $30 \mathrm{mg}$. Ten months after administration of afatinib, recurrence of the tumor on the primary site was reported. Bronchoscopic re-biopsy of the tissue was performed; pathologically, the tumor was a typical squamous cell carcinoma with alveolar structures. On immunohistochemical examination, the tumor was positive for 440 (Fig. 2) and negative for thyroid transcription factor (TTF)-1 and Napsin A. Although the levels of CYFRA were increased $(10.2 \mathrm{ng} / \mathrm{ml})$ at this point, the levels of CEA remained within normal limits. Paraffin sections were analyzed to determine the presence of an acquired resistance mutation. In addition to the exon 19 deletion originally harbored by the tumor at the time of diagnosis, this analysis detected the T790M mutation. The patient received treatment with the third-generation EGFR-TKI osimertinib (80 mg), and a partial response was observed in the recurrent site. The levels of CYFRA returned to normal, and the patient was relapse-free 1 year after treatment initiation. At the last follow up in April 2018, the patient was symptom free.

\section{Discussion}

Numerous studies have reported the use of re-biopsy in NSCLC patients with disease progression following first- or second-generation EGFR-TKI therapy $(3,4)$. Moreover, recent studies have reported squamous cell transformation in patients with NSCLC during the course of treatment (5-18), described as a mechanism of acquired resistance to these agents.

In the present case, the T790M mutation was detected in combination with squamous cell transformation in a patient with lung adenocarcinoma who was treated with afatinib. The tumor maintained the original exon 19 deletion, suggesting a monoclonal origin and clonal progression. Administration of osimertinib resulted in a partial response and was well-tolerated, without severe adverse events. Third-generation EGFR-TKIs, 


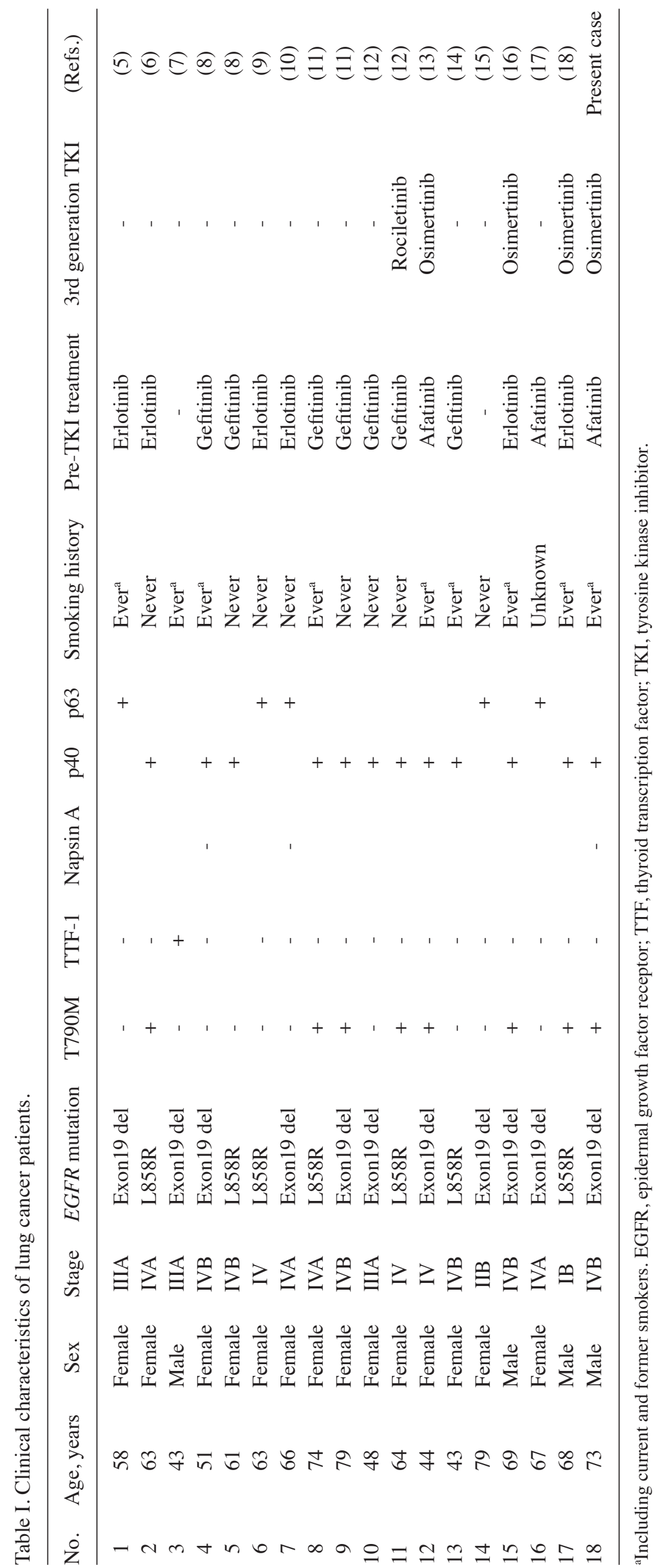


such as osimertinib and rociletinib, selectively block T790M mutant clones. The efficacy of osimertinib in the treatment of T790M-positive NSCLC has been demonstrated (19). As shown in Table I, a total of 18 cases of squamous cell transformation in lung adenocarcinoma harboring an EGFR-activating mutation after treatment with cytotoxic chemotherapy or EGFR-TKIs have been reported (5-18); in 8 of those, the T790M mutation was also detected. These patients were treated with EGFR-TKIs as first-line therapy. There was no significant association of the T790M mutation with age, gender, clinical stage, smoking history or activating mutation status (data not shown). Five of the patients with the T790M mutation received treatment with third-generation EGFR-TKIs, and the recurrence site improved in all cases. EGFR-TKIs have been reported to be less effective in the treatment of non-adenocarcinoma tumors compared with adenocarcinoma (20). However, third-generation EGFR-TKIs may be beneficial in the treatment of squamous cell carcinoma harboring the T790M mutation.

The pathogenesis of squamous cell transformation in lung adenocarcinoma remains elusive. In NSCLC, the presence of adenocarcinoma and squamous cell carcinoma is associated with distinct clinical characteristics, gene expression, and specific biomarkers. Adenocarcinoma usually arises in the distal airway, whereas squamous cell carcinoma is more frequently reported in the proximal airway. EGFR mutations are more frequent in adenocarcinoma compared with squamous cell carcinoma. Immunohistochemical analysis in adenocarcinoma demonstrated diffusely positive staining for TTF-1 and Napsin A, and negative staining for p40 and p63 expression, whereas the opposite was observed in squamous cell carcinoma. In the present study, detection of an EGFR exon19 deletion in the re-biopsy specimen decreased the possibility of de novo squamous cell carcinoma development. There are two possible mechanisms of transformation: The first hypothesis is that adenocarcinoma may develop squamous metaplasia, an authentic transformation during therapy. In a mouse model, liver kinase B1 inactivation resulted in accumulation of reactive oxygen species (ROS), eventually causing progressive transdifferentiation of the adenocarcinoma to squamous cell carcinoma through an intermediate mixed status (21). Although excessive levels of ROS induce apoptosis and decrease cell proliferation in adenocarcinoma, squamous cell carcinoma is strongly resistant to ROS. The second hypothesis is that the initial tumor contains squamous cell carcinoma components in varying proportions, clinically termed adenosquamous carcinoma. This is a rare subtype of NSCLC ( $\leq 4 \%$ of all lung carcinomas), defined by mixed adenocarcinomatous and squamous cell carcinomatous components $>10 \%$ (22). In some cases, clinically defined adenocarcinoma or squamous cell carcinoma, diagnosed through biopsy or cytology specimens, may in fact be adenosquamous carcinoma. Therefore, it is important todiagnose adenosquamous carcinoma using surgical specimens rather than biopsy samples. The clinicopathological characteristics of adenosquamous carcinoma remain unclear. It has been reported that adenosquamous carcinoma is more aggressive and has a worse prognosis compared with either adenocarcinoma or squamous cell carcinoma $(23,24)$. By contrast, several studies revealed similar frequencies of EGFR mutations between adenosquamous carcinoma and adenocarcinoma $(25,26)$, an association between adenosquamous carcinoma with $E G F R$-activating mutations, and good response to EGFR-TKIs (27).

The aforementioned hypotheses suggest that resistant clones, which consist of squamous cell carcinoma, are increased through EGFR-TKI therapy. As shown in Fig. 1, the CEA and CYFRA tumor markers were elevated at the time of diagnosis and returned within normal limits simultaneously during therapy with afatinib. The levels of CEA, which is particularly associated with adenocarcinoma, remained within the normal range, whereas the level of CYFRA, a tumor marker for follow-up of squamous cell carcinoma, increased to $10.2 \mathrm{ng} / \mathrm{ml}$ during disease progression. This suggests that the initial adenocarcinoma was diminished, and the squamous cell carcinoma component was limited by treatment with afatinib, a finding consistent with the latter hypothesis. However, it is possible that adenocarcinoma progressively transdifferentiates into squamous cell carcinoma during therapy, even if the original tumor contains both adenocarcinoma and squamous cell carcinoma.

In the present case, the T790M mutation was identified in a patient with lung adenocarcinoma and presence of squamous cell carcinoma, as shown by re-biopsy. The patient received osimertinib treatment, with an evident beneficial effect on the recurrence site. These findings suggest that this third-generation EGFR-TKI is a viable treatment option for patients with lung adenocarcinoma exhibiting squamous cell transformation harboring the T790M mutation. Re-sequencing is warranted, regardless of the detection of morphological transition of primary adenocarcinoma. The use of re-biopsy may also be important for investigating the progression of lung cancer and overcoming resistance to therapeutic agents.

\section{Acknowledgements}

Not applicable.

\section{Funding}

No funding was received.

\section{Availability of data and materials}

The datasets generated and/or analyzed during the present study are available from the corresponding author on reasonable request.

\section{Authors' contributions}

FY, EK, AW and YS examined and cared for the patient. FY wrote the manuscript. EK, AW and YS contributed to the writing of the manuscript and commented at all stages. FY had full access to all the data in the study and has final responsibility for the decision to submit for publication. All the authors have read and approved the final version of this manuscript.

\section{Ethics approval and consent to participate}

Official approval for gene analysis was obtained in advance from the Ethics Committee for Genomic Research at Showa 
University (approval no. 113). The patient provided written informed consent for gene analysis.

\section{Patient consent for publication}

Written informed consent was obtained from the patient for publication of the case details and any associated images.

\section{Competing interests}

All the authors declare that they have no conflict of interest in connection with this study. The authors received no payment or services from a third party in relation to this study.

\section{References}

1. Yu HA, Arcila ME, Rekhtman N, Sima CS, Zakowski MF Pao W, Kris MG, Miller VA, Ladanyi M and Riely GJ: Analysis of tumor specimens at the time of acquired resistance to EGFR-TKI therapy in 155 patients with EGFR-mutant lung cancers. Clin Cancer Res 19: 2240-2247, 2013.

2. Cancer Therapy Evaluation Program (CTEP) Common Terminology Criteria for Adverse Events (CTCAE), Version 4.0 National Cancer Institute, 2010.

3. Chouaid C, Dujon C, Do P, Monnet I, Madroszyk A, Le Caer H, Auliac JB, Berard H, Thomas P, Lena H, et al: Feasibility and clinical impact of re-biopsy in advanced non small-cell lung cancer: A prospective multicenter study in a real-world setting. Lung Cancer 86: 170-173, 2014.

4. Nosaki K, Satouchi M, Kurata T, Yoshida T, Okamoto I, Katakami N, Imamura F, Tanaka K, Yamane Y, Yamamoto N, et al: Re-biopsy status among non-small cell lung cancer patients in Japan: A retrospective study. Lung Cancer 101: 1-8, 2016.

5. Scher KS, Saldivar JS, Fishbein M, Marchevsky A and Reckamp KL: EGFR-mutated lung cancer with T790M-acquired resistance in the brain and histologic transformation in the lung. J Natl Compr Canc Netw 11: 1040-1044, 2013.

6. Bugano DDG, Kalhor N, Zhang J, Neskey M and William WN: Squamous-cell transformation in a patient with lung adenocarcinoma receiving erlotinib: Co-occurrence with T790M mutation. Cancer Treat Commun 4: 34-36, 2015.

7. Burkart J, Shilo K, Zhao W, Ozkan E, Ajam A and Otterson GA: Metastatic squamous cell carcinoma component from an adenosquamous carcinoma of the lung with identical epidermal growth factor receptor mutations. Case Rep Pulmonol 2015: 283875, 2015.

8. Hsieh MS, Jhuang JY, Hua SF and Chou YH: Histologic evolution from adenocarcinoma to squamous cell carcinoma after gefitinib treatment. Ann Thorac Surg 99: 316-319, 2015.

9. Kuiper JL, Ronden MI, Becker A, Heideman DA, van Hengel P, Ylstra B, Thunnissen E and Smit EF: Transformation to a squamous cell carcinoma phenotype of an EGFR-mutated NSCLC patient after treatment with an EGFR-tyrosine kinase inhibitor. J Clin Pathol 68: 320-321, 2015.

10. Levin PA, Mayer M, Hoskin S, Sailors J, Oliver DH and Gerber DE: Histologic transformation from adenocarcinoma to squamous cell carcinoma as a mechanism of resistance to EGFR inhibition. J Thorac Oncol 10: e86-e88, 2015.

11. Jukna A, Montanari G, Mengoli MC, Cavazza A, Covi M, Barbieri F, Bertolini F and Rossi G: Squamous cell carcinoma 'transformation' concurrent with secondary T790M mutation in resistant EGFR-mutated adenocarcinomas. J Thorac Oncol 11: e49-e51, 2016

12. Haratani K, Hayashi H, Watanabe S, Kaneda H, Yoshida T, Takeda M, Shimizu T and Nakagawa K: Two cases of EGFR mutation-positive lung adenocarcinoma that transformed into squamous cell carcinoma: Successful treatment of one case with rociletinib. Ann Oncol 27: 200-202, 2016.
13. Bruno R, Proietti A, Alì G, Puppo G, Ribechini A, Chella A and Fontanini G: Squamous cell transformation and EGFR T790M mutation as acquired resistance mechanisms in a patient with lung adenocarcinoma treated with a tyrosine kinase inhibitor: A case report. Oncol Lett 14: 5947-5951, 2017.

14. Longo L, Mengoli MC, Bertolini F, Bettelli S, Manfredini S and Rossi G: Synchronous occurrence of squamous-cell carcinoma 'transformation' and EGFR exon 20 S768I mutation as a novel mechanism of resistance in EGFR-mutated lung adenocarcinoma. Lung Cancer 103: 24-26, 2017.

15. Le T, Sailors J, Oliver DH, Mayer M, Hoskin S and Gerber DE: Histologic transformation of EGFR mutant lung adenocarcinoma without exposure to EGFR inhibition. Lung Cancer 105: 14-16, 2017.

16. Okabe N, Takagi H, Mine H, Fukai S, Minemura H and Suzuki H: Osimertinib for epidermal growth factor receptor mutation-positive lung adenocarcinoma that transformed to T790M-positive squamous cell carcinoma. J Thorac Oncol 12: e167-e169, 2017.

17. Clery E, Pisapia P, Feliciano S, Vigliar E, Marano A, De Luca C, Malapelle U, Troncone $\mathrm{G}$ and Bellevicine C: There is still a role for cytology in the 'liquid biopsy' era. A lesson from a TKI-treated patient showing adenocarcinoma to squamous cell carcinoma transition during disease progression. J Clin Pathol 70: 798-802, 2017.

18. Izumi H, Yamasaki A, Ueda Y, Sumikawa T, Maeta H, Nakamoto $S$ and Shimizu E: Squamous cell carcinoma transformation from EGFR-mutated lung adenocarcinoma: A case report and literature review. Clin Lung Cancer 19: e63-e66, 2018.

19. Jänne PA, Yang JC, Kim DW, Planchard D, Ohe Y, Ramalingam SS, Ahn MJ, Kim SW, Su WC, Horn L, et al: AZD9291 in EGFR inhibitor-resistant non-small-cell lung cancer. N Engl J Med 372: 1689-1699, 2015.

20. Shukuya T, Takahashi T, Kaira R, Ono A, Nakamura Y, Tsuya A, Kenmotsu H, Naito T, Kaira K, Murakami H, et al: Efficacy of gefitinib for non-adenocarcinoma non-small-cell lung cancer patients harboring epidermal growth factor receptor mutations: A pooled analysis of published reports. Cancer Sci 102: 1032-1037, 2011.

21. Hou S, Zhou S, Qin Z, Yang L, Han X, Yao S and Ji H: Evidence, mechanism, and clinical relevance of the transdifferentiation from lung adenocarcinoma to squamous cell carcinoma. Am J Pathol 187: 954-962, 2017.

22. Travis WD, Brambilla E, Nicholson AG, Yatabe Y, Austin JHM, Beasley MB, Chirieac LR, Dacic S, Duhig E, Flieder DB, et al: The 2015 world health organization classification of lung tumors: Impact of genetic, clinical and radiologic advances since the 2004 classification. J Thorac Oncol 10: 1243-1260, 2015.

23. Shimizu J, Oda M, Hayashi Y, Nonomura A and Watanabe Y: A clinicopathologic study of resected cases of adenosquamous carcinoma of the lung. Chest 109: 989-994, 1996.

24. Gawrychowski J,Bruliński K, Malinowski Eand Papla B: Prognosis and survival after radical resection of primary adenosquamous lung carcinoma. Eur J Cardiothorac Surg 27: 686-692, 2005.

25. Jia XL and Chen G: EGFR and KRAS mutations in Chinese patients with adenosquamous carcinoma of the lung. Lung Cancer 74: 396-400, 2011.

26. Wang R, Pan Y, Li C, Zhang H, Garfield D, Li Y, Ye T, Hu H, Luo X, Li H, et al: Analysis of major known driver mutations and prognosis in resected adenosquamous lung carcinomas. J Thorac Oncol 9: 760-768, 2014.

27. Fan L, Yang H, Yao F, Zhao Y, Gu H, Han $\mathrm{K}$ and Zhao H: Clinical outcomes of epidermal growth factor receptor tyrosine kinase inhibitors in recurrent adenosquamous carcinoma of the lung after resection. Onco Targets Ther 10: 239-245, 2017. 